\title{
Ultrathin Highly Luminescent Two-Monolayer Colloidal CdSe Nanoplatelets
}

\author{
Savas Delikanli, Guannan Yu, Aydan Yeltik, Sumanta Bose, Talha Erdem, Junhong Yu, \\ Onur Erdem, Manoj Sharma, Vijay Kumar Sharma, Ulviyya Quliyeva, Sushant Shendre, \\ Cuong Dang, Dao Hua Zhang, Tze Chien Sum, Weijun Fan, and Hilmi Volkan Demir*
}

Surface effects in atomically flat colloidal CdSe nanoplatelets (NLPs) are significantly and increasingly important with their thickness being reduced to subnanometer level, generating strong surface related deep trap photoluminescence emission alongside the bandedge emission. Herein, colloidal synthesis of highly luminescent two-monolayer (2ML) CdSe NPLs and a systematic investigation of carrier dynamics in these NPLs exhibiting broad photoluminescence emission covering the visible region with quantum yields reaching $90 \%$ in solution and $85 \%$ in a polymer matrix is shown. The astonishingly efficient Stokes-shifted broadband photoluminescence $(P L)$ emission with a lifetime of $\approx 100$ ns and the extremely short PL lifetime of around $0.16 \mathrm{~ns}$ at the bandedge signify the participation of radiative midgap surface centers in the recombination process associated with the underpassivated Se sites. Also, a proof-of-concept hybrid LED employing $2 \mathrm{ML}$ CdSe NPLs is developed as color converters, which exhibits luminous efficacy reaching $300 \mathrm{Im} \mathrm{W}_{\mathrm{opt}^{-1}}$. The intrinsic absorption of the $2 \mathrm{ML}$ CdSe NPLs $\left(\approx 2.15 \times 10^{6} \mathrm{~cm}^{-1}\right)$ reported in this study is significantly larger than that of CdSe quantum dots $\left(\approx 2.8 \times 10^{5} \mathrm{~cm}^{-1}\right)$ at their first exciton signifying the presence of giant oscillator strength and hence making them favorable candidates for next-generation light-emitting and light-harvesting applications.

\section{Introduction}

2D materials have recently attracted a great deal of interest owing to their exceptional electronic structure and optical characteristics originating from their highly anisotropic thin structure. ${ }^{[1-3]}$ Their 2D structure offers superior conductivity in the lateral dimension compared to the other colloidal nanostructures since carriers can move freely in plane without encountering boundaries or barriers, which makes them advantageous for novel electronic and optoelectronic devices. ${ }^{[1,4]}$ Colloidal 2D semiconductor nanosheets and nanoplatelets (NPLs) have been successfully synthesized with thickness control at the monolayer level in various compositions including $\mathrm{CdSe}^{[2,5]}$ and $\mathrm{PbS}^{[6]}$ by means of chemical routes. CdSe NPLs were shown to exhibit extraordinary properties such as extremely narrow electroluminescence emission with full-width at half-maximum (FWHM) of around
Dr. S. Delikanli, J. Yu, Dr. M. Sharma, Dr. V. K. Sharma, S. Shendre, Prof. C. Dang, Prof D. H. Zhang, Prof. W. Fan, Prof. H. V. Demir Luminous! Center of Excellence for Semiconductor Lighting and Displays

School of Electrical and Electronic Engineering

Nanyang Technological University

50 Nanyang Avenue, Singapore 639798, Singapore

E-mail: hvdemir@ntu.edu.sg

Dr. S. Delikanli, Dr. G. Yu, Dr. V. K. Sharma, Prof. T. C. Sum,

Prof. H. V. Demir

Division of Physics and Applied Physics

School of Physical and Mathematical Sciences

Nanyang Technological University

21 Nanyang Link, Singapore 639798, Singapore

Dr. S. Delikanli, Dr. A. Yeltik, Dr. T. Erdem, O. Erdem, Dr. M. Sharma,

Dr. V. K. Sharma, U. Quliyeva, Prof. H. V. Demir

Department of Electrical and Electronics Engineering

Department of Physics

UNAM - Institute of Materials Science and Nanotechnology

Bilkent University

Ankara 06800, Turkey

The ORCID identification number(s) for the author(s) of this article can be found under https://doi.org/10.1002/adfm.201901028.

\author{
Dr. G. Yu \\ Shenzhen Key Laboratory of Two-Dimensional Materials \\ and Devices (SKTMD) \\ SZU-NUS Collaborative Innovation Center for Optoelectronic Science \\ and Technology, and Key Laboratory of Optoelectronic Devices \\ and Systems of Ministry of Education and Guangdong Province \\ Shenzhen University \\ Shenzhen 518060, China \\ Dr. S. Bose, Prof. W. Fan \\ Optimus \\ Centre for Optoelectronics and Biophotonics \\ School of Electrical and Electronic Engineering \\ Nanyang Technological University \\ 50 Nanyang Avenue, Singapore 639798, Singapore \\ Prof D. H. Zhang \\ Nanophotonics Laboratory \\ School of Electrical and Electronic Engineering \\ Nanyang Technological University \\ Singapore 639798, Singapore
}

DOI: $10.1002 / \mathrm{adfm} .201901028$ 
$12 \mathrm{~nm},{ }^{[7]}$ the state-of-the-art amplified spontaneous emission (ASE) thresholds ${ }^{[8,9]}$ and highly anisotropic emission. ${ }^{[3]}$ In addition, these quasi-2D quantum wells (QWs) provide a convenient platform for the design of novel complex hetero-architectures because of the opportunity of growing a purely lateral crown layer as well as a shell coating layer with an atomic layer precision. ${ }^{[10-16]}$ Specifically, 4ML and 5ML CdSe NPLs having thicknesses larger than $1 \mathrm{~nm}$ have gained the greatest attention because of their high QYs (as high as 80\%) and extremely narrow photoluminescence (PL) emission originating from the recombination of the excitons at the bandedge. ${ }^{[2,17]}$

As the thickness of these colloidal 2D semiconductors is further reduced to subnanometer level, surface induced effects start to dominate similar to the CdSe QDs having diameter of $<2 \mathrm{~nm}$, and lead to a strong broadband deep trap emission along with the bandedge emission, ${ }^{[13,18-20]}$ which can also be exploited to achieve white light emission. Broad Stokes-shifted emission, which grows stronger with decreasing nanocrystal size, ${ }^{[21]}$ has been extensively studied in ultrasmall colloidal CdSe nanocrystals and was attributed to the midgap states originating from selenium (Se) dangling bonds owing to the unpassivated Se atoms at the surface. ${ }^{[20,22,23]}$ In these strongly confined systems, bandedge emission is due to the recombination of the delocalized electron-hole pair, the exciton, while their broadband deep trap emission is mediated through recombination of a trapped hole with the delocalized electron. ${ }^{[22]}$ However, QY of CdSe QDs having diameter of $2 \mathrm{~nm}<$ was found to be generally low, ranging from a few percents to $45 \%$ after surface treatment techniques, ${ }^{[24]}$ which undermines their exploitation in optoelectronic applications. In CdSe NPLs, the emission from surface related states becomes dominant only for the NPLs thinner than four monolayers, which corresponds to thickness of $1.2 \mathrm{~nm} \cdot{ }^{[18]}$ From subnanometer thick NPLs, 3ML CdSe NPLs have been investigated in a few articles and was shown to exhibit strong deep trap state emission with QY of $\approx 1 \%-30 \%,{ }^{[25,26]}$ which is considerably lower than QY of the thicker NPLs $\approx 30 \%-70 \%$, ${ }^{[17]}$ while 2ML CdSe NPLs have been largely excluded from the previous studies possibly because of their poor optical properties due to uncontrolled synthesis and their anomalous optical properties. ${ }^{[27]}$ However, 2D 2ML CdSe NPLs present potentially an excellent platform for optoelectronic applications and investigation of their carrier dynamics owing to their significantly strong pure quantum confinement and extremely anisotropic 2D structure.

In this study, we present the colloidal synthesis and systematic optical investigation of highly efficient 2ML CdSe NPLs having intrinsic absorption cross-section significantly larger than thicker CdSe NPLs (thickness $>1 \mathrm{~nm}$ ) and QDs, which indicates the manifestation of giant oscillator strength (GOST). PL kinetics in these 2ML CdSe NPLs can be significantly altered by varying the amounts of the utilized Se precursor. Addition of excess Se leads to stronger Stokes-shifted broad emission and significant increase in QY as well, which can be attributed to the astonishingly efficient emission from the recombination of electrons with the trapped holes localized at unpassivated Se sites because of extremely large exciton binding energy and superior in-plane conductivity. Carrier dynamics were further investigated by time-resolved PL measurements and the observed significantly long PL lifetime ( $\geq 100 \mathrm{~ns}$ ) from the broadband emission is attributed to the decreased spatial overlap between the wave functions of the electrons and the trapped holes. In addition, we explored the performance of these NPLs by integrating their solid films with a near ultraviolet-light emitting diode (UV-LED). The luminous efficacy of the obtained integrated hybrid LED reaches $\approx 300 \mathrm{~lm} \mathrm{~W}_{\text {opt }}^{-1}$ as a result of the strong overlap of the emission with the human eye sensitivity function and exceptionally high QY of our NPL solids.

\section{Results and Discussion}

We synthesized 2ML CdSe NPLs by making use of an acetate salt for enabling the lateral growth of the NPLs. Details of the syntheses are presented in the Supporting Information. Lateral size of the 2ML CdSe NPLs can be controlled by adjusting the growth time after the injection of the Se precursor (trioctylphosphine selenide) as can be seen from the transmission electron microscopy (TEM) images given in Figure 1a-e. CdSe NPLs having lateral sizes from 100 to $>10000 \mathrm{~nm}^{2}$ can be grown easily by just changing the growth time. As the lateral size of these NPLs grows larger, they are rolled up to form tubular forms as can be seen from the TEM images. A high-resolution TEM image of a rolled NPL tube having a length of $\approx 150 \mathrm{~nm}$ is shown in the inset of Figure 1e. The absorption spectra of the 2ML CdSe NPLs presented in Figure 1f, which correspond to the NPLs shown in Figure 1a-e, reveal an anomalous dependence of the strength of different excitonic transitions on the lateral size of the NPLs. The sharp transitions located around 372 and $393 \mathrm{~nm}$ are associated with the light and heavy hole transitions, respectively, in these 2ML CdSe NPLs. ${ }^{[27,28]}$ These 2ML CdSe NPLs consists of three monolayers of $\mathrm{Cd}$ and two monolayers of Se with both surfaces of NPLs ending predominantly with Cd layer. ${ }^{[28]}$ As can be deduced from Figure 1f, the relative strength of the heavy hole and light hole $(372 \mathrm{~nm})$ transitions largely depends on the lateral size of the 2ML CdSe NPLs, even leading to almost complete quenching of heavy hole transition for the smallest NPLs. Such dependence of light and heavy hole transitions on the lateral size will be explored elsewhere. As the lateral size of the 2ML CdSe NPLs is increased, the heavy hole transition dominates as can be seen in Figure 1f, similar to the observations on thicker NPLs. ${ }^{[2]}$

The intrinsic absorption spectrum of the 2ML CdSe NPLs corresponding to the NPLs shown in Figure 1e, which exhibits an absorption spectrum similar to other thicker CdSe NPLs in terms of the relative strength of heavy hole and light hole transitions, is presented in Figure 1g. For the calculation of absorption crosssection, we followed the method used in our previous work ${ }^{[29]}$ and intrinsic absorption cross-section was calculated by dividing the obtained absorption cross-section by the volume of the NPLs. The intrinsic absorption of these CdSe NPLs $\left(\approx 2.15 \times 10^{6} \mathrm{~cm}^{-1}\right)$ at their first excitonic peak is significantly larger than that of other thicker CdSe NPLs $\left(\approx 4.1 \times 10^{5} \mathrm{~cm}^{-1}\right)$ and CdSe QDs $\left(\approx 2.8 \times 10^{5} \mathrm{~cm}^{-1}\right)^{[27]}$ making them advantageous for light-harvesting applications which require strong light-matter interactions. The increase in the intrinsic absorption in this system is expected compared to thicker nanocrystals because of the higher optical field penetration due to the extremely large aspect ratio ${ }^{[27]}$ and reduction of the dielectric factor leading higher exciton binding energy. ${ }^{[2]}$ 

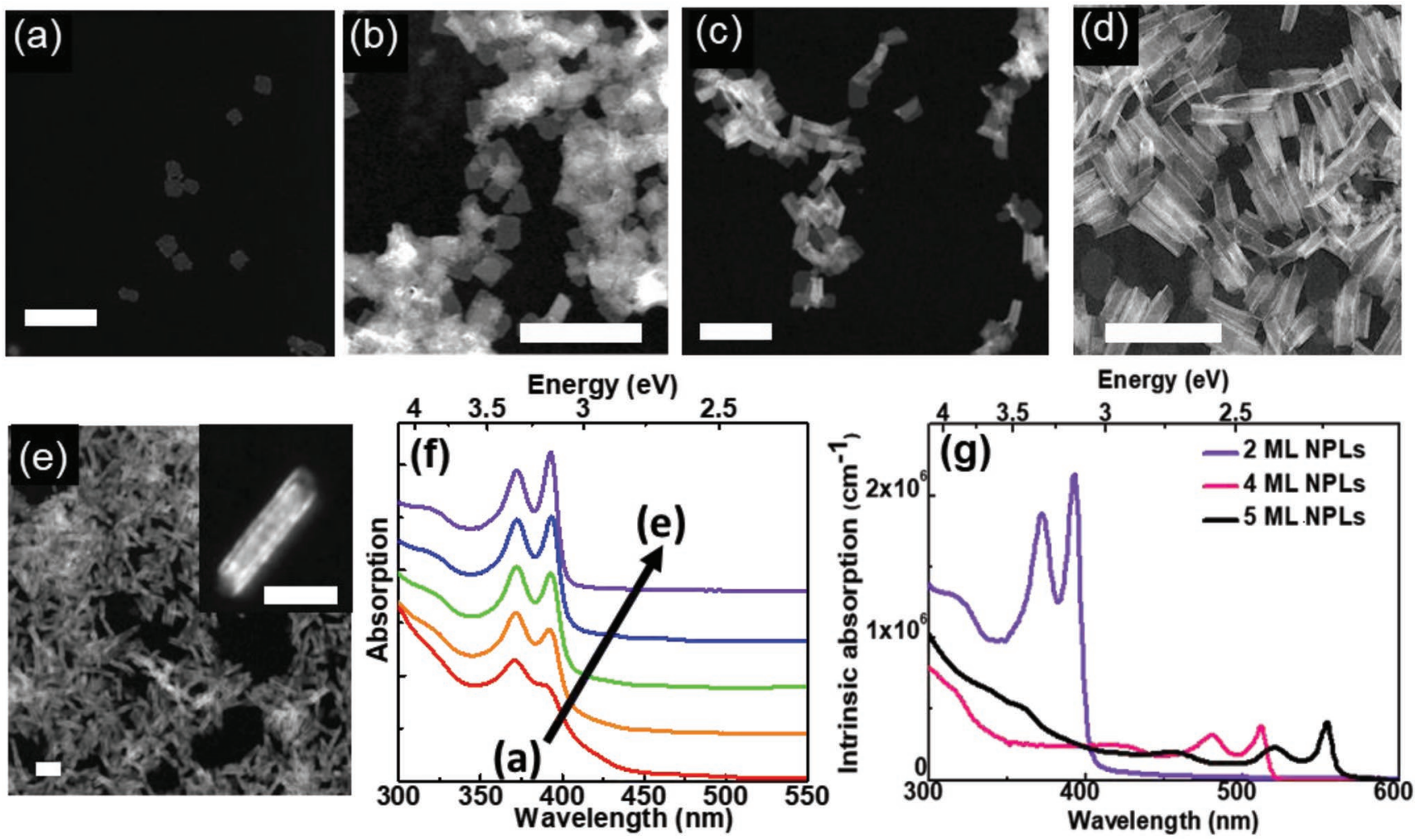

Figure 1. a-e) TEM images of $2 M L$ CdSe NPLs at different sizes. Inset of (e) shows a high-resolution image of a single NPL. The scale bar for every TEM image is $100 \mathrm{~nm}$. f) Absorption spectra of the corresponding NPLs shown in insets (a-e).g) Intrinsic absorption spectra of NPLs having thickness of $2 \mathrm{ML}, 4 \mathrm{ML}$, and $5 \mathrm{ML}$.

To investigate the surface-assisted emission kinetics in these NPLs, we studied continuous-wave PL (CW-PL) emission characteristics of two different samples synthesized using excess cadmium (Cd) and excess Se. Synthesis of CdSe NPLs generally requires inclusion of excess amount of cadmium acetate precursor to promote 2D growth and assure capping of the outermost surface layer with Cd atoms and NPLs synthesis with excess Cd approach leads to PL emission with narrow emission associated with the recombination of the carriers at the bandedge according to the previous reports. ${ }^{[2]}$ PL spectrum of $2 \mathrm{ML}$ CdSe NPLs grown by excess Cd shows strong narrow emission at $\approx 405 \mathrm{~nm}$ together with broad emission at $\approx 550 \mathrm{~nm}$ with FWHM of $\approx 150 \mathrm{~nm}$ as presented in Figure 2 a. QY of these 2ML NPLs synthesized with excess Cd is relatively low $<20 \%$, which is similar to that of $3 \mathrm{ML}$ CdSe NPLs. ${ }^{[25,26]}$ To the best of our knowledge, there is no previous report on the QY of $2 \mathrm{ML}$ CdSe NPLs to compare in the literature. On the other hand, 2ML CdSe NPLs synthesized using excess amount of Se exhibit almost purely broadband PL emission centered at $\approx 535 \mathrm{~nm}$ with a FWHM of $\approx 150 \mathrm{~nm}$ as shown in Figure 2 a and a QY of $90 \%$ in solution. QY measurements were executed by following the procedure developed by De Mello et al. at an excitation wavelength of $380 \mathrm{~nm} \cdot{ }^{[30,31]}$ We performed PL excitation (PLE) spectroscopy to probe the origin of the broadband emission. The PLE spectra of the NPLs taken in the broadband emission (600 and $500 \mathrm{~nm}$ ) show clearly the excitonic peaks associated with the heavy and light hole transitions as presented in Figure 3a. The sharp bandedge absorption and independence of PLE spectrum on the emission wavelength suggest that samples are monodisperse and the emission (both bandedge and broadband deep trap emission) are not from a mixture of NPLs having different thicknesses.

The emission at $\approx 405 \mathrm{~nm}$, which is only a few nanometer Stokes-shifted from the heavy hole transition, is clearly a result of excitonic recombination at the bandedge while the Stokesshifted broadband emission can be attributed to the surface related midgap states as these states become dominant recombination centers in small nanocrystals $(2 \mathrm{~nm}<$ diameter) as well. ${ }^{[20,21]}$ The high PL QY accompanied with strong Stokesshifted broadband emission indicates that the midgap states are radiative. A strong increase in the activity of surface states is expected in these ultrathin NPLs as a consequence of the extremely large surface-to-volume ratio. In these subnanometer thick NPLs, roughly $40 \%$ of the atoms are located at the surface. In addition, the increase of the broadband emission in the PL spectrum with the addition of excess Se suggests that these emission centers are associated with the unpassivated Se sites and hence trapping of the generated holes as reported in CdSe QDs. ${ }^{[20,32]}$ According to the earlier results, surface Se atoms in CdSe nanocrystals are unpassivated and leads to deep trap emission in these structures. ${ }^{[22,32-34]}$

To investigate the existence of surface Se atoms, we performed X-ray photoelectron spectroscopy (XPS) measurements on our samples. The high-resolution XPS spectra of Se 3d orbital for the NPLs synthesized with excess Cd and excess Se are shown in Figure 4a,b, respectively. For the XPS analysis, 
(a)
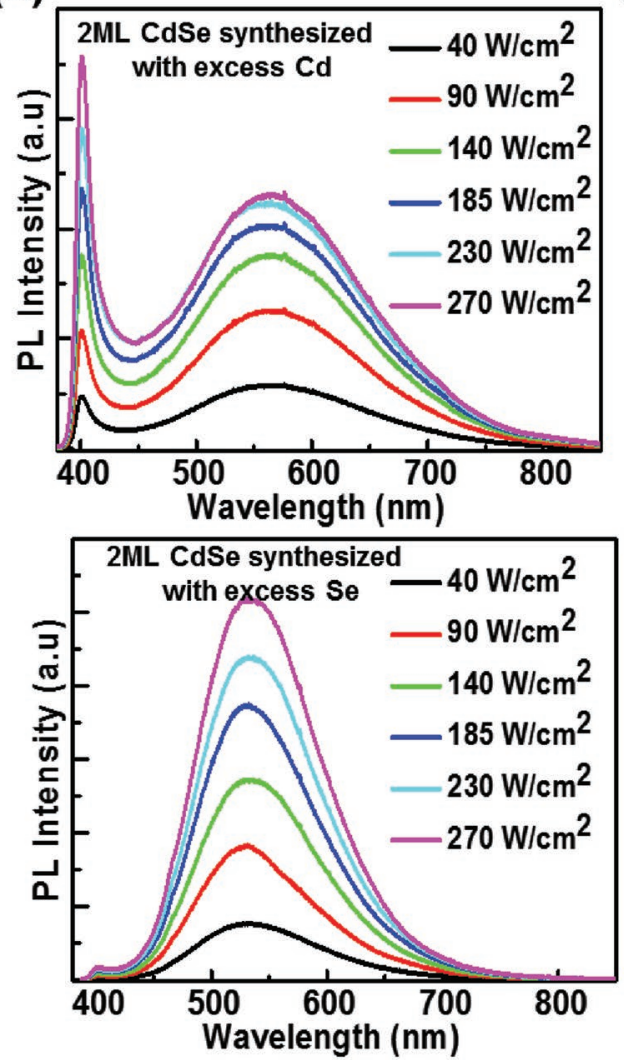

(b)
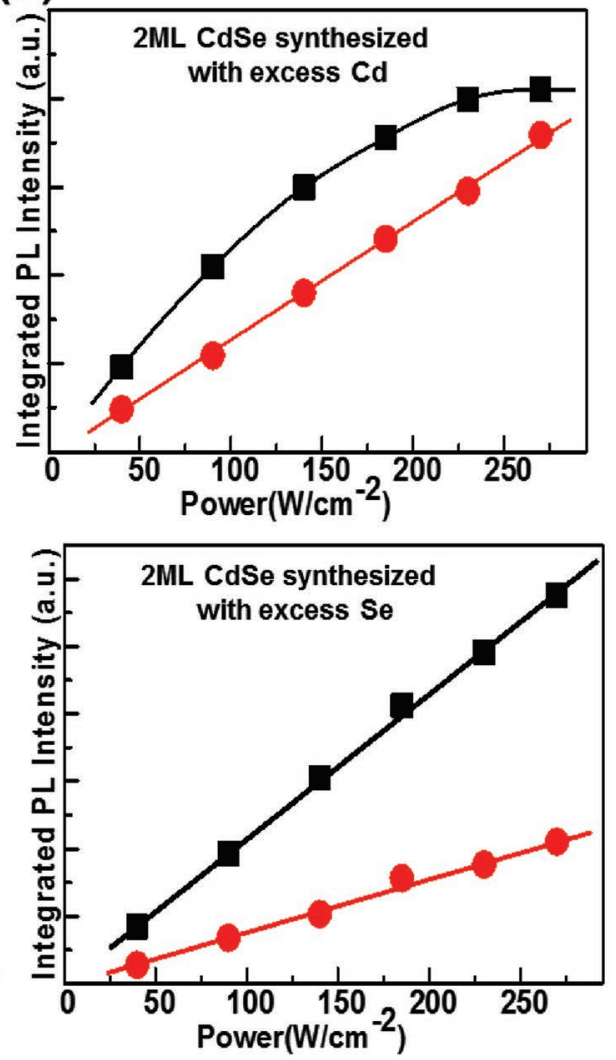

Figure 2. a) Power dependent PL spectra of $2 \mathrm{ML}$ CdSe NPLs synthesized with excess Cd and excess Se. b) Power dependence of the integrated PL intensity of the bandedge (red) and surface emission center (black) of 2ML CdSe NPLs synthesized with excess Cd and excess Se.

the C1s peak is referenced at $285 \mathrm{eV}$. In both the cases, we can resolve the XPS peak into three peaks positioning at 55.3, 54.5 , and $53.6 \mathrm{eV}$. The peaks at the 55.3 and $54.5 \mathrm{eV}$ are associated with the Se $3 d$ doublet, i.e., Se $3 d_{5 / 2}$ and $3 d_{3 / 2}$ regions as reported earlier in bulk CdSe. The lower energy peak at $53.6 \mathrm{eV}$ is significantly more pronounced in the sample synthesized with excess Se compared to the sample synthesized with excess $\mathrm{Cd}$. We attribute this lower energy peak to the unpassivated Se atoms at the surface having a reduced number of neighboring $\mathrm{Cd}$ atoms. ${ }^{[35]}$ The pronounced peak at $53.6 \mathrm{eV}$ side observed in the sample synthesized with excess Se suggests that this sample has a significantly large number of unpassivated Se atoms located at surface. This has been further confirmed by the XPS elemental analysis showing higher Se (36 at\%) concentration in the sample synthesized with excess Se in comparison to the sample synthesized with excess $\mathrm{Cd}(\mathrm{Se} \approx 32$ at\%).

To further investigate origin of the efficient broadband emission in these subnanometer thick NPLs, CdS shell, and CdS crown were grown on these NPLs. PL, absorption, and PLE spectra of the CdSe core-only, CdSe/CdS core/crown, and
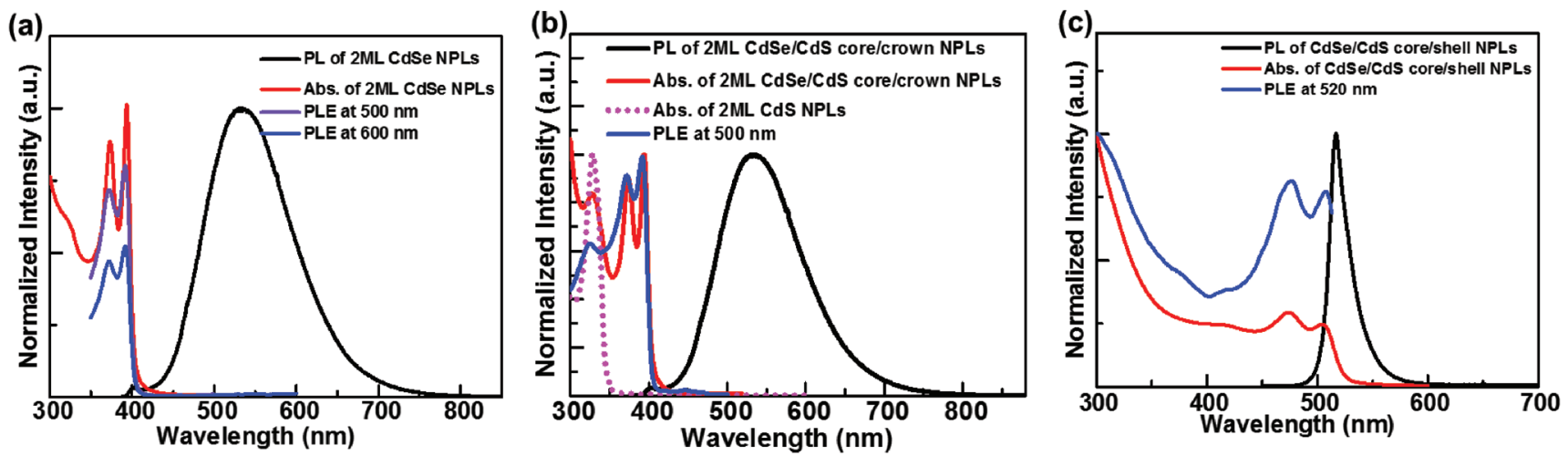

Figure 3. PL, absorption, and PLE spectra of a) CdSe core-only, b) CdSe/CdS core/crown, and c) CdSe/CdS core/shell NPLs. 

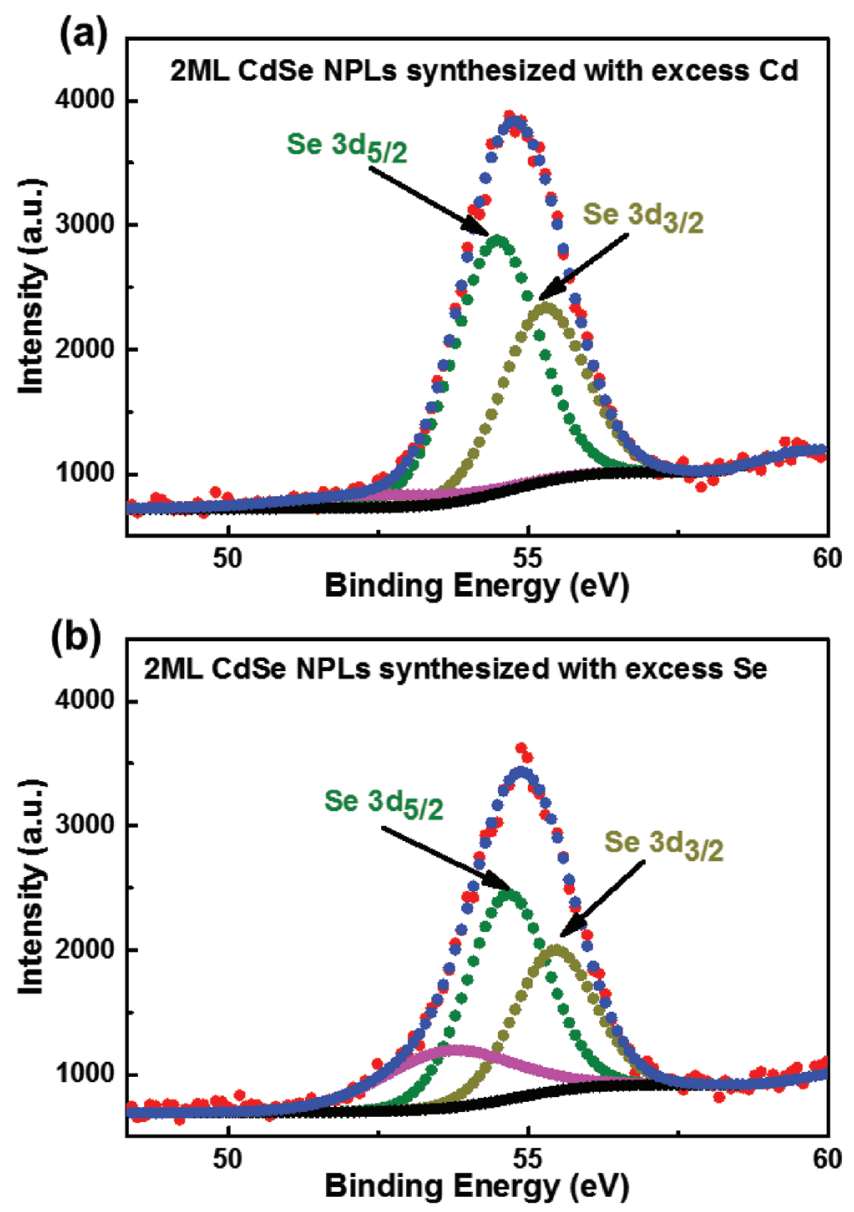

Figure 4. High-resolution XPS spectrum of Se 3d orbital obtained for $2 M L$ CdSe NPLs synthesized with excess amount of a) Cd and b) Se. Red circles represent the actual data while blue circles represent the sum of the fitted peaks and background signal (black circles). 2ML CdSe NPLs synthesized with excess Se show pronounced peak appearing (magenta circles) at the lower binding energy, which can be attributed to the presence of unpassivated Se at the surface.

CdSe/CdS core/shell NPLs are presented in Figure 3a-c, respectively. The peak appearing at $\approx 325 \mathrm{~nm}$ in absorption and PLE spectra with the growth of crown is associated with the grown CdS layer as 2ML CdS NPLs exhibits a strong transition at the same spectral position as also shown in Figure 3b. ${ }^{[2]}$ For the shell growth, we deposited 2ML of CdS shell (one monolayer to each side) on our 2ML CdSe NPLs using colloidal atomic layer deposition technique. ${ }^{[12]}$ The strong red shift of heavy and light hole transitions (>100 nm) observed in the absorption spectrum demonstrates the successful growth of $2 \mathrm{ML}$ CdS shell on the 2ML CdSe NPLs. The PL spectrum of the core/crown sample is identical to that of the core-only sample while the PL from the core/shell sample is considerably narrow (FWHM $\approx 20 \mathrm{~nm}$ ) and Stokes-shifted by only $\approx 5 \mathrm{~nm}$ from the heavy hole transition, which is similar to the previous results on core/shell samples based on thicker NPLs. ${ }^{[12]}$ A few nanometer Stokes-shifted narrow emission from the core/shell sample signifies that the midgap surface states associated with the efficient broadband greenish emission is passivated with the addition of shell and hence this broadband emission observed in these NPLs indeed originates from recombination centers at the surface of the NPLs. However, these core/shell NPLs show significantly lower QYs (QY $\approx 10 \%-15 \%$ ) compared to $2 \mathrm{ML}$ CdSe NPLs emitting through almost only from the surface centers. The low QY in this core/shell system is associated with the shallow hole trapping sites leading nonradiative recombination. ${ }^{[36,37]}$ In addition, the insensitivity of the PL spectra to the passivation of CdSe core edges with a wide bandgap CdS crown suggests that broadband deep trap emission is extensively associated with radiative recombination centers located at the lateral surfaces rather than the peripheral ones playing a significant role in the recombination process and trapping of the carriers as previously demonstrated in layered 2D perovskites. ${ }^{[38]}$

The Stokes-shifted emission also in some cases can be attributed to the self-trapped excitons (STE) associated with the strong electron-phonon coupling. ${ }^{[39,40]}$ In the case of STEs, due to the strong electron-phonon coupling, the peak intensity of the PL increases linearly with the excitation intensity without any saturation or deviation till the photodegradation of the material, ${ }^{[40-42]}$ while the PL emission originating from defect sites can be saturated or can deviate from linear behavior under high excitation intensities due to the limited number of permanent defect sites. ${ }^{[43]}$ Figure 2a shows the excitation power density (up to $\approx 300 \mathrm{~W} \mathrm{~cm} \mathrm{~cm}^{-2}$ ) dependence of PL emission from 2ML CdSe NPLs dispersed in hexane solution. We estimated the integrated PL intensity of the bandedge emission by fitting the sharp bandedge emission to a Gaussian function in each PL curve. The integrated emission from surface centers was then calculated by subtracting the obtained bandedge emission from the entire PL emission spectrum. The power dependence of the integrated PL intensity of the bandedge and surface centers in 2ML CdSe NPLs is shown in Figure 2b. For the sample having strong bandedge emission together with the defect emission, the emission from defect sites saturates at high excitation power densities $\left(\approx 250 \mathrm{~W} \mathrm{~cm}^{-2}\right)$ while the emission from bandedge keeps increasing almost linearly as can be seen in Figure $2 \mathrm{a}, \mathrm{b}$. This can be explained by saturation of the defect sites at higher excitation powers in this sample having relatively low number of defect sites. However, for the sample having almost purely broad emission from surface emission centers, the broad emission rises linearly with increasing excitation power density $>300 \mathrm{~W} \mathrm{~cm}^{-2}$ indicating that the existence of high number of defect sites, which remain unsaturated while exposed to similar excitation powers when the sample was grown by excess $\mathrm{Cd}$.

We performed time-resolved PL measurements on $2 \mathrm{ML}$ CdSe NPLs in solution to probe the carrier dynamics using a streak camera and a laser source with the excitation wavelength of $350 \mathrm{~nm}$ (repetition rate: $1 \mathrm{kHz}$, pulse width: $150 \mathrm{fs}$, power: 1-15 $\mu \mathrm{W})$. PL decay curves of the NPLs at the bandedge emission are shown in Figure 5a. PL decay is approximately three orders-of-magnitude faster at the bandedge with a lifetime of $\approx 150-300$ ps compared to the PL decay at the broadband emission having a lifetime of $\geq 100 \mathrm{~ns}$. PL decay is multiexponential in NPLs according to the previous reports because of the multichannel radiative and nonradiative pathways. ${ }^{[44]}$ We employed a triexponential decay function for the fitting of the PL decay curves measured at the bandedge emission. The PL lifetime components are given in Table S1 in the Supporting Information. The intensity averaged lifetime of the PL emission at 

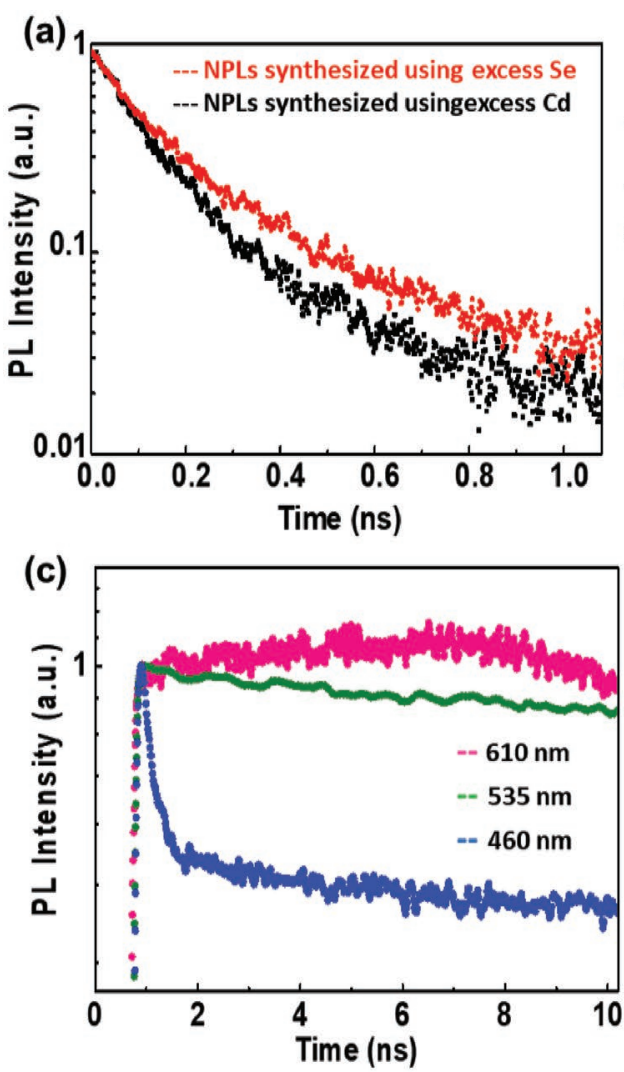

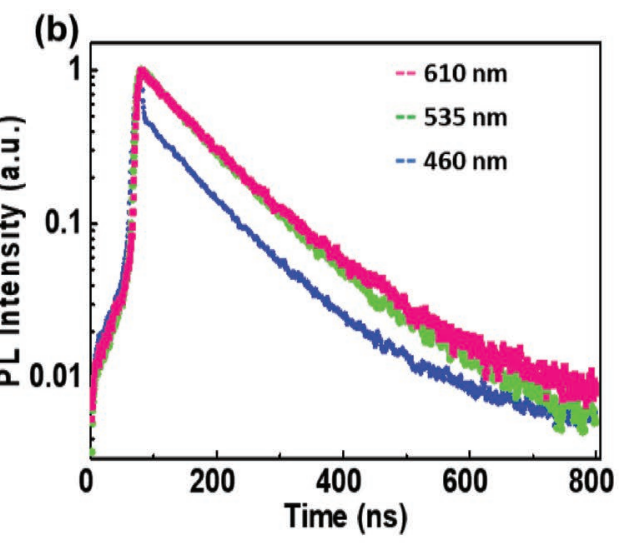

(d)

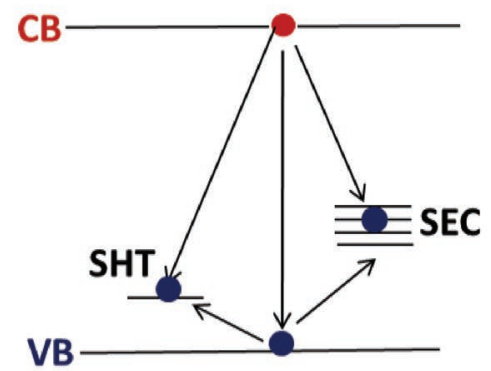

Figure 5. a) PL decay curves of the NPLs at the bandedge emission. b,c) PL decay curves of the NPLs at different wavelengths around surface center emission. Inset (c) shows the PL decay kinetics at the earlier times while inset (b) depicts PL decay on a longer window. d) Schematic representation of the carrier recombination pathways in these NPLs. Red (blue) dots correspond to photogenerated electrons (holes). As depicted, carriers can recombine at the bandedge, surface emission centers (SECs), or shallow hole traps (SHT) mediated by structural defects.

the bandedge $(405 \mathrm{~nm})$ is around 0.16 and $0.27 \mathrm{~ns}$ for the sample synthesized with excess Se and excess $\mathrm{Cd}$, respectively. These measured PL lifetimes are significantly shorter than the previously reported PL decay lifetimes of 1-4 ns for 4ML and $5 \mathrm{ML}$ CdSe NPLs, ${ }^{[2,45]}$ which can be attributed to the significantly strong and fast carrier trapping in these subnanometer thick NPLs. We associate the two shorter lifetimes, $T_{1} \approx 80-120 \mathrm{ps}$ and $T_{2} \approx 300 \mathrm{ps}$, to the trapping of the carriers and the longer component having a lifetime around $0.6 \mathrm{~ns}$ to the recombination of excitons at the bandedge. ${ }^{[36]}$ In addition, the lifetime $(<1 \mathrm{~ns})$ of the $2 \mathrm{ML}$ CdSe NPLs at the bandedge is shorter compared to the lifetime of several nanoseconds observed in 5ML CdSe NPLs at room temperature. ${ }^{[36,44]}$

PL decay curves at the high energy side $(460 \mathrm{~nm})$, the peak $(535 \mathrm{~nm})$, and the lower energy side $(610 \mathrm{~nm})$ of the broadband emission for the sample having strong Stokes-shifted emission are given in Figure 5b-c. The PL decay at the high energy side has a short lifetime component $(\approx 200 \mathrm{ps})$ together with a long lifetime component of $\approx 100$ ns while intensity of the PL at the low energy side exhibits a slight increase in the first few nanoseconds as can be seen in Figure 5b. We also observed similar decay kinetics from the sample having stronger bandedge emission. This quick decrease in the PL intensity at the high energy side together with the increase in the PL intensity within first few ns at the low energy side can be attributed to the relaxation of holes via releasing of phonons within the trap site before they recombine radiatively with the electrons and relaxation of photogenerated carriers into low lying defect sites present within the same NPL. The redshift of PL emission with time also reveals this energy relaxation as shown in Figure S3 in the Supporting Information. Here, the carriers trapped at the surface are holes as indicated by the dependence of CW-PL characteristics to the addition of excess Se and very similar lifetimes of hole trapping at the surface sites previously reported. ${ }^{[46]}$ Significantly longer lifetime of Stokesshifted broadband emission can be explained by the decrease in the spatial overlap between the wavefunctions of delocalized electrons and holes localized at the noncoordinated Se sites at the surface. PL decay at the Stokes-shifted emission exhibits a monoexponential decay after first few nanoseconds with a long lifetime of $\approx 100 \mathrm{~ns}$, which is almost two ordersof-magnitude longer than the previously reported PL lifetimes from CdSe NPLs. ${ }^{[2]}$ Here, trapped holes at the surface centers can encounter the delocalized electrons and can recombine radiatively facilitated by their $2 \mathrm{D}$ structure which allows the electrons to move freely within the plane and also the strong Coulomb interaction due to the low dielectric constant in these NPLS. In addition, the relaxation of carriers into the midgap surface states suppresses their trapping by nonradiative defect sites.

The higher QY of the sample emitting almost purely through surface centers with only $<1 \%$ contribution from 

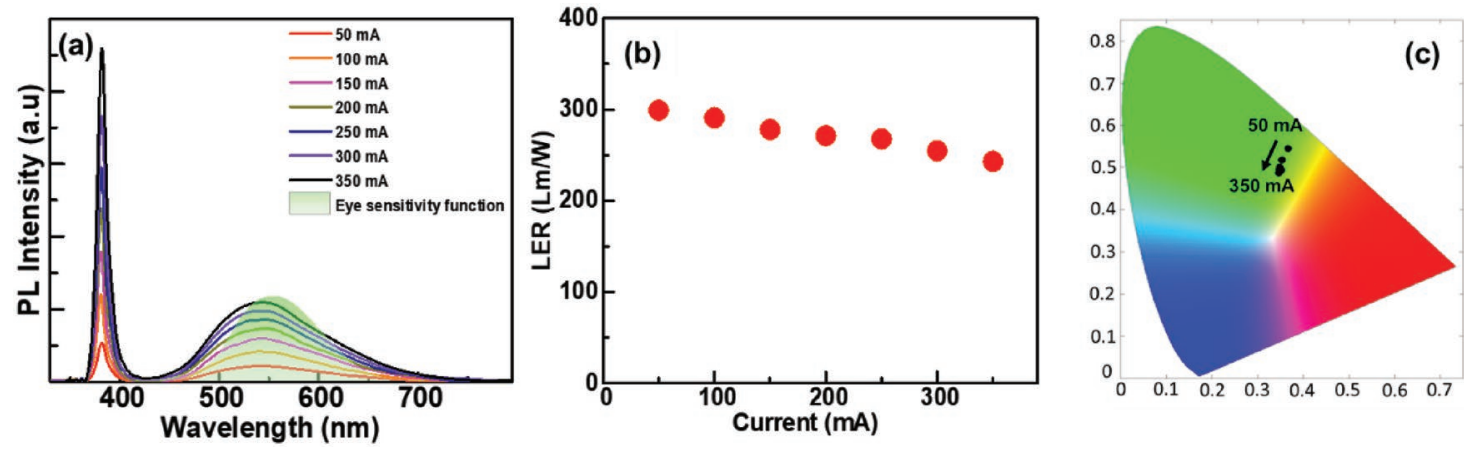

Figure 6. a) Emission spectra, b) optical luminous efficacy of the NPL integrated hybrid LED at various driving currents, and c) Chromaticity coordinates of the hybrid LED at various driving currents.

bandedge (as shown in Figure 2c) can be explained by trapping of the holes strongly by the surface centers emitting efficiently. This stronger trapping leads to significantly shorter PL lifetime at the bandedge $(\approx 0.16 \mathrm{~ns})$ compared to that of sample having stronger bandedge emission $(\approx 0.27 \mathrm{~ns})$ at the bandedge. We attribute PL decay component with $T_{3} \approx 300 \mathrm{ps}$ to the hole trapping leading to predominantly nonradiative recombination in CdSe NPLs as similar lifetimes $(\approx 300 \mathrm{ps})$ associated hole trapping leading nonradiative recombination were reported in NPLs. ${ }^{[36]}$ Additionally, the existence of nonradiative hole trapping channel not only in the core CdSe and but also in the core/shell CdSe/CdS NPLs ${ }^{[36]}$ indicates this nonradiative channel is associated with structural defects rather than surface centers. The illustration of the recombination of the photogenerated carriers in these NPLs is depicted in Figure 5d, where carriers can recombine at the bandedge, surface trap sites, or shallow hole traps mediated by structural defects.

Motivated by the optically superior performance of the NPLs having Stokes-shifted emission, we explored their photoluminescence capability by integrating solid films of these NPLs (obtained by using PMMA) with a near UV-LED emitting at $380 \mathrm{~nm}$. The details of the film formation and optical measurements are given in the Supporting Information. The QY of their solid films was measured as $85 \%$. The exceptionally high QY of the solid film of these NPLs is as a result of large Stokes-shifted emission reducing the losses associated with the reabsorption and Förster resonance energy transfer as also demonstrated in solid films of type-II NPLs. ${ }^{[4]}$ This NPL integrated LED was driven between 50 and $350 \mathrm{~mA}$ and the obtained emission spectra were presented in Figure 6a. Chromaticity coordinates of the LED were calculated between $(0.37,0.54)$ and $(0.35,0.49)$ with increasing current from 50 to $350 \mathrm{~mA}$ (Figure 6c). The luminous efficacy of optical radiation (LER) of this LED reaches $300 \mathrm{~lm} \mathrm{~W}_{\mathrm{opt}}^{-1}$ as presented in Figure 6b, greatly surpassing the previous results from the LEDs utilizing NPLs ${ }^{[48]}$ and small QDs $(2 \mathrm{~nm}<\text { diameter })^{[49,50]}$ because of the strong overlap of the NPL emission with the human eye sensitivity function and the high QY of solid films of our NPLs. However, as can be seen in Figure $6 \mathrm{~b}$, the emission from the exciting LED at $380 \mathrm{~nm}$ leads to a reduction at high currents in the LER performance since it can be only weakly sensed by the human eye. This white LED also has a low color rendering index (CRI) around 50 indicating a poor reproduction of the real colors of the objects. The main reason behind this is the lack of a blue color component in our LED despite the presence of green, yellow, and red portions of the visible spectrum. The color rendition performance of our device could be improved by addition of a blue-emitting color component and then our LED made of 2ML CdSe NPLs can be a promising candidate for efficient quality solid-state lighting. Our proof-of-concept demonstration in here shows that 2ML CdSe NPLs are highly promising for efficient solid-state lighting in green/yellow with LER reaching

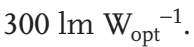

\section{Conclusion}

In summary, we demonstrated the synthesis of highly efficient 2ML CdSe NPLs and studied recombination dynamics of the photogenerated carriers within these 2D nanostructures through systematic investigation by continuous-wave and timeresolved PL techniques. Surface emission centers mediated by the localized holes at unpassivated Se sites provide a pathway for radiative recombination assisted by large in-plane mobility and exciton binding energy in these 2D nanostructures. The relaxation of photocarriers into the midgap surface centers suppresses their capture by nonradiative centers likely associated with structural defect sites. The nearly monoexponential decay and extremely long lifetime of these surface midgap states reveal the radiative nature of these states together with the QYs reaching $\approx 90 \%$ for the NPLs having almost entirely pure broad greenish emission. The NPLs film integrated LED exhibits luminous efficacy of 250-300 $\mathrm{lm} \mathrm{W}_{\text {opt }}{ }^{-1}$, considerably outperforming those previously attained using NPLs and small QDs, and hence indicating their potential in solid-state lighting in green/yellow window. Our results show that these highly efficient NPLs can serve as an outstanding platform for next-generation light-generating and -harvesting applications particularly considering their extremely large intrinsic absorption cross-section and efficient Stokes-shifted emission.

\section{Supporting Information}

Supporting Information is available from the Wiley Online Library or from the author. 


\section{Acknowledgements}

This research is supported by the National Research Foundation, Prime Minister's Office, Singapore under its NRF Investigatorship Award program (NRF-NRFI2016-08) and the Singapore Agency for Science, Technology and Research (A*STAR) SERC Pharos Program under Grant No. 15273 00025. T.C.S. acknowledges the funding support from the Ministry of Education (MOE) Tier 1 grant RG 173/16, and MOE Tier 2 grant MOE2016-T2-1-034; and from the Singapore National Research Foundation through the Competitive Research Programme NRF-CRP14-2014-03. C.D. would like to acknowledge the financial support from Singapore Ministry of Education AcRF Tier-1 grant (MOERG178/17). W.J.F. would like to thank funding support from Singapore MOE AcRF Tier 1 RG87/17. G.Y. acknowledges China Postdoctoral Science Foundation 2017M620385. H.V.D., also gratefully acknowledges the support from TUBA and EURYI. O.E. acknowledges support from TUBITAK BIDEB.

\section{Conflict of Interest}

The authors declare no conflict of interest.

\section{Keywords}

CdSe nanoplatelets, colloidal, giant oscillator strength, high quantum yield, ultrathin

Received: February 1, 2019 Revised: May 27, 2019

Published online: July 4, 2019

[1] K. S. Novoselov, A. K. Geim, S. V. Morozov, D. Jiang, Y. Zhang, S. V. Dubonos, I. V. Grigorieva, A. A. Firsov, Science 2004, 306, 666.

[2] S. Ithurria, M. D. Tessier, B. Mahler, R. P. S. M. Lobo, B. Dubertret, A. L. Efros, Nat. Mater. 2011, 10, 936.

[3] R. Scott, J. Heckmann, A. V. Prudnikau, A. Antanovich, A. Mikhailov, N. Owschimikow, M. Artemyev, J. I. Climente, U. Woggon, N. B. Grosse, A. W. Achtstein, Nat. Nanotechnol. 2017, 12, 1155.

[4] M. Nasilowski, B. Mahler, E. Lhuillier, S. Ithurria, B. Dubertret, Chem. Rev. 2016, 116, 10934

[5] J. Joo, J. S. Son, S. G. Kwon, J. H. Yu, T. Hyeon, J. Am. Chem. Soc. 2006, 128, 5632.

[6] C. Schliehe, B. H. Juarez, M. Pelletier, S. Jander, D. Greshnykh, M. Nagel, A. Meyer, S. Foerster, A. Kornowski, C. Klinke, H. Weller, Science 2010, 329, 550.

[7] F. Fan, P. Kanjanaboos, M. Saravanapavanantham, E. Beauregard, G. Ingram, E. Yassitepe, M. M. Adachi, O. Voznyy, A. K. Johnston, G. Walters, G.-H. Kim, Z.-H. Lu, E. H. Sargent, Nano Lett. 2015, 15, 4611.

[8] B. Guzelturk, Y. Kelestemur, M. Olutas, S. Delikanli, H. V. Demir, ACS Nano 2014, 8, 6599.

[9] C. She, I. Fedin, D. S. Dolzhnikov, P. D. Dahlberg, G. S. Engel, R. D. Schaller, D. V. Talapin, ACS Nano 2015, 9, 9475.

[10] M. D. Tessier, P. Spinicelli, D. Dupont, G. Patriarche, S. Ithurria, B. Dubertret, Nano Lett. 2014, 14, 207.

[11] Y. Kelestemur, M. Olutas, S. Delikanli, B. Guzelturk, M. Z. Akgul, H. V. Demir, J. Phys. Chem. C 2015, 119, 2177.

[12] S. Ithurria, D. V. Talapin, J. Am. Chem. Soc. 2012, 134, 18585.

[13] S. Delikanli, M. Z. Akgul, J. R. Murphy, B. Barman, Y. Tsai, T. Scrace, P. Zhang, B. Bozok, P. L. Hernández-Martínez, J. Christodoulides,
A. N. Cartwright, A. Petrou, H. V. Demir, ACS Nano 2015, 9, 12473.

[14] F. Muckel, S. Delikanli, P. L. Hernández-Martínez, T. Priesner, S. Lorenz, J. Ackermann, M. Sharma, H. V. Demir, G. Bacher, Nano Lett. 2018, 18, 2047.

[15] S. Delikanli, B. Guzelturk, P. L. Hernández-Martínez, T. Erdem, Y. Kelestemur, M. Olutas, M. Z. Akgul, H. V. Demir, Adv. Funct. Mater. 2015, 25, 4282.

[16] Y. Kelestemur, B. Guzelturk, O. Erdem, M. Olutas, K. Gungor, H. V. Demir, Adv. Funct. Mater. 2016, 26, 3570.

[17] M. Olutas, B. Guzelturk, Y. Kelestemur, A. Yeltik, S. Delikanli, H. V. Demir, ACS Nano 2015, 9, 5041.

[18] S. Ithurria, B. Dubertret, J. Am. Chem. Soc. 2008, 130, 16504.

[19] J. C. Newton, K. Ramasamy, M. Mandal, G. K. Joshi, A. Kumbhar, R. Sardar, J. Phys. Chem. C 2012, 116, 4380.

[20] M. J. Bowers, J. R. McBride, S. J. Rosenthal, J. Am. Chem. Soc. 2005, $127,15378$.

[21] C. F. Landes, M. Braun, M. A. El-Sayed, J. Phys. Chem. B 2001, 105, 10554.

[22] D. F. Underwood, T. Kippeny, S. J. Rosenthal, J. Phys. Chem. B 2001, 105, 436

[23] J. Chem. Phys. 1994, 100, 2831.

[24] T. E. Rosson, S. M. Claiborne, J. R. McBride, B. S. Stratton, S. J. Rosenthal, J. Am. Chem. Soc. 2012, 134, 8006.

[25] M. Sharma, K. Gungor, A. Yeltik, M. Olutas, B. Guzelturk, Y. Kelestemur, T. Erdem, S. Delikanli, J. R. McBride, H. V. Demir, Adv. Mater. 2017, 29, 1700821.

[26] Z. Li, X. Peng, J. Am. Chem. Soc. 2011, 133, 6578.

[27] A. W. Achtstein, A. Antanovich, A. Prudnikau, R. Scott, U. Woggon, M. Artemyev, J. Phys. Chem. C 2015, 119, 20156

[28] A. Riedinger, F. D. Ott, A. Mule, S. Mazzotti, P. N. Knüsel, S. J. P. Kress, F. Prins, S. C. Erwin, D. J. Norris, Nat. Mater. 2017, 16, 743.

[29] A. Yeltik, S. Delikanli, M. Olutas, Y. Kelestemur, B. Guzelturk, H. V. Demir, J. Phys. Chem. C 2015, 119, 26768.

[30] J. C. de Mello, H. F. Wittmann, R. H. Friend, Adv. Mater. 1997, 9, 230.

[31] T. Erdem, Z. Soran-Erdem, V. K. Sharma, Y. Kelestemur, M. Adam, N. Gaponik, H. V. Demir, Nanoscale 2015, 7, 17611.

[32] A. C. Carter, C. E. Bouldin, K. M. Kemner, M. I. Bell, J. C. Woicik, S. A. Majetich, Phys. Rev. B 1997, 55, 13822.

[33] X. Wang, L. Qu, J. Zhang, X. Peng, M. Xiao, Nano Lett. 2003, 3, 1103.

[34] A. M. Smith, S. Nie, Acc. Chem. Res. 2010, 43, 190.

[35] A. Veamatahau, B. Jiang, T. Seifert, S. Makuta, K. Latham, M. Kanehara, T. Teranishi, Y. Tachibana, Phys. Chem. Chem. Phys. $2015,17,2850$.

[36] L. T. Kunneman, J. M. Schins, S. Pedetti, H. Heuclin, F. C. Grozema, A. J. Houtepen, B. Dubertret, L. D. A. Siebbeles, Nano Lett. 2014, 14, 7039 .

[37] J. R. Murphy, S. Delikanli, T. Scrace, P. Zhang, T. Norden, T. Thomay, A. N. Cartwright, H. V. Demir, A. Petrou, Appl. Phys. Lett. 2016, 108, 242406.

[38] J. C. Blancon, H. Tsai, W. Nie, C. C. Stoumpos, L. Pedesseau, C. Katan, M. Kepenekian, C. M. M. Soe, K. Appavoo, M. Y. Sfeir, S. Tretiak, P. M. Ajayan, M. G. Kanatzidis, J. Even, J. J. Crochet, A. D. Mohite, Science 2017, 355, 1288.

[39] T. Hu, M. D. Smith, E. R. Dohner, M.-J. Sher, X. Wu, M. T. Trinh, A. Fisher, J. Corbett, X. Y. Zhu, H. I. Karunadasa, A. M. Lindenberg, J. Phys. Chem. Lett. 2016, 7, 2258.

[40] E. R. Dohner, A. Jaffe, L. R. Bradshaw, H. I. Karunadasa, J. Am. Chem. Soc. 2014, 136, 13154.

[41] Z. Yuan, C. Zhou, Y. Tian, Y. Shu, J. Messier, J. C. Wang, L. J. van de Burgt, K. Kountouriotis, Y. Xin, E. Holt, K. Schanze, R. Clark, T. Siegrist, B. Ma, Nat. Commun. 2017, 8, 14051. 
[42] A. Yangui, D. Garrot, J. S. Lauret, A. Lusson, G. Bouchez, E. Deleporte, S. Pillet, E. E. Bendeif, M. Castro, S. Triki, Y. Abid, K. Boukheddaden, J. Phys. Chem. C 2015, 119, 23638.

[43] M. B. Teunis, K. N. Lawrence, P. Dutta, A. P. Siegel, R. Sardar, Nanoscale 2016, 8, 17433.

[44] M. D. Tessier, C. Javaux, I. Maksimovic, V. Loriette, B. Dubertret, ACS Nano 2012, 6, 6751.

[45] W. Cho, S. Kim, I. Coropceanu, V. Srivastava, B. T. Diroll, A. Hazarika, I. Fedin, G. Galli, R. D. Schaller, D. V. Talapin, Chem. Mater. 2018, 30, 6957.
[46] S. L. Sewall, R. R. Cooney, K. E. H. Anderson, E. A. Dias, D. M. Sagar, P. Kambhampati, J. Chem. Phys. 2008, 129, 084701.

[47] B. Liu, S. Delikanli, Y. Gao, D. Dede, K. Gungor, H. V. Demir, Nano Energy 2018, 47, 115.

[48] Y. Kelestemur, B. Guzelturk, O. Erdem, M. Olutas, T. Erdem, C. F. Usanmaz, K. Gungor, H. V. Demir, J. Phys. Chem. C 2017, 121, 4650.

[49] J. D. Gosnell, S. J. Rosenthal, S. M. Weiss, IEEE Photonics Technol. Lett. 2010, 22, 541.

[50] M. A. Schreuder, J. D. Gosnell, N. J. Smith, M. R. Warnement, S. M. Weiss, S. J. Rosenthal, J. Mater. Chem. 2008, 18, 970. 